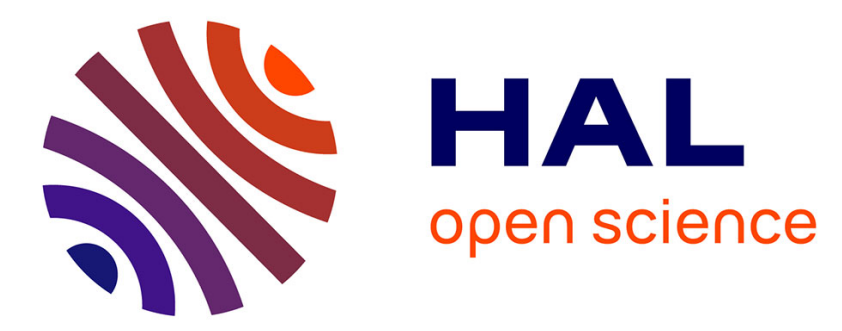

\title{
Transversal stability of the bouncing ball on a concave surface
}

J.-y Chastaing, G Pillet, N Taberlet, J.-C Géminard

\section{To cite this version:}

J.-y Chastaing, G Pillet, N Taberlet, J.-C Géminard. Transversal stability of the bouncing ball on a concave surface. Physical Review E: Statistical, Nonlinear, and Soft Matter Physics, 2015, 91, pp.052918. 10.1103/PhysRevE.91.052918 . hal-01158683

\section{HAL Id: hal-01158683 \\ https://hal.science/hal-01158683}

Submitted on 11 Jun 2015

HAL is a multi-disciplinary open access archive for the deposit and dissemination of scientific research documents, whether they are published or not. The documents may come from teaching and research institutions in France or abroad, or from public or private research centers.
L'archive ouverte pluridisciplinaire HAL, est destinée au dépôt et à la diffusion de documents scientifiques de niveau recherche, publiés ou non, émanant des établissements d'enseignement et de recherche français ou étrangers, des laboratoires publics ou privés. 


\title{
Transversal stability of the bouncing ball on a concave surface
}

\author{
J.-Y. Chastaing, ${ }^{1}$ G. Pillet, ${ }^{1}$ N. Taberlet, ${ }^{1,2}$ and J.-C. Géminard ${ }^{1}$ \\ ${ }^{1}$ Université de Lyon, Laboratoire de Physique, Ecole Normale Supérieure de Lyon, CNRS, 46 Allée d'Italie, 69364 Lyon cedex 07, France \\ ${ }^{2}$ Université de Lyon, UFR de Physique, Université Claude Bernard Lyon I, 69100 Villeurbanne, France
}

(Received 29 January 2015; published 27 May 2015)

\begin{abstract}
A ball bouncing repeatedly on a vertically vibrating surface constitutes the famous "bouncing ball" problem, a nonlinear system used in the 1980s, and still in use nowadays, to illustrate the route to chaos by period doubling. In experiments, in order to avoid the ball escape that would be inevitable with a flat surface, a concave lens is often used to limit the horizontal motion. However, we observe experimentally that the system is not stable. The ball departs from the system axis and exhibits a pendular motion in the permanent regime. We propose theoretical arguments to account for the decrease of the growth rate and of the asymptotic-size of the trajectory when the frequency of the vibration is increased. The instability is very sensitive to the physics of the contacts, which makes it a potentially interesting way to study the collisions rules, or to test the laws used in numerical studies of granular matter.
\end{abstract}

DOI: 10.1103/PhysRevE.91.052918

PACS number(s): 05.45.Ac, 45.50.Tn, 45.70.-n, 46.32.+x

\section{INTRODUCTION}

Studies of vibrated grains are numerous in literature [1]. The problem has attracted the attention of engineers and physicists for both practical and fundamental reasons. In practice, mechanical vibrations are likely to rearrange the grains and, for instance, to lead to changes in the density of granular assemblies [1-4], to convection [5], to grain separation [6,7], to subharmonic instabilities [8,9], etc. The problem has also attracted the attention of the physicists as the mechanical vibrations can also be considered as a way to introduce a "temperature" in an, otherwise, athermal system [10,11]. Looking for minimal systems they considered, in particular, layers of grains covering partially a flat and horizontal surface that is vibrated vertically. Such systems, with grains consisting of spherical beads, were regarded as prototypes of two-dimensional granular fluids that revealed nonstandard velocity fluctuations [12] and phase transitions [13,14]. These works were extended to elongated particles making the systems resemble liquid crystals [15]. Asymmetric particles can self-propel and the system becomes a prototype of active matter [16]. The case of a single particle bouncing freely upon a vibrating surface has also attracted much attention, first as an example of nonlinear system exhibiting a transition to chaos by period doubling $[17,18]$. Authors also considered the statistical properties of the particle in the chaotic regime, which can be viewed as a simplified one-dimensional gas [19] or a dissipative system maintained in a permanent regime [20].

The experimental realization of the so-called "bouncing ball" problem requires the use of a trick to impose the motion of the ball along the vertical. Indeed, any misalignment of the vibrating surface leads the ball to migrate in the, almost, horizontal plane and, finally, to escape the system. The use of a guiding vertical tube is a possibility [21], but it introduces undesired friction with the vertical walls that is potentially annoying. The usual trick is to use a concave surface instead of a flat surface [17]. The expectation is that the slope repels the bead toward the lowest point when the ball departs from center. Even if the technique has been successfully used in many experimental studies, the stability of the motion of the ball along the vertical is not ensured. Apart from a numerical study reporting an intermittent lateral motion due to asperities of the surface in contact [22], the problem has not been tackled. In the present article, we report experimental evidence that the system is, in fact, unstable, and we propose a theoretical approach to identify the underlying mechanisms.

\section{EXPERIMENTAL SETUP AND PROTOCOL}

The experimental setup (Fig. 1) consists of a parabolic concave lens of radius of curvature $R=15.6 \mathrm{~cm}$ at the center (external diameter $10 \mathrm{~cm}$ ) driven into a sinusoidal motion along the vertical by means of an electromagnetic shaker (Brüel\&Kjaer, Type V406 M4). A steel bead of radius $r=0.5 \mathrm{~cm}$ is bouncing upon it. The powerful shaker makes possible the use of a mobile part (lens and holder) of large inertia, which avoids any significant receding of the bottom surface due to the impact with the bead. The power amplifier (Kepco, BOP50-4M) driving the shaker receives a sinusoidal signal from an analog output of a data acquisition board (National Instruments, PXIe 1073). In the present study, the typical frequency $f$ of the vibration ranges from 30 to $70 \mathrm{~Hz}$, for a resulting peak acceleration never greater than $0.7 g$, where $g$ denotes the acceleration due to gravity. A noncontact inductive sensor (Electrocorp, EMD1053, sensing range $0-3 \mathrm{~mm}$, resolution $1 \mu \mathrm{m}$ ) is used to measure the vertical displacement $z(t)$ of the lens, which is thus of about a few tens of $\mu \mathrm{m}$ and never exceeds $500 \mu \mathrm{m}$. In addition, a microphone (Sennheiser, MKH20 P48), plugged into a soundcard (TASCAM, US 122MKII) connected to the computer, is used to detect the bead-lens collisions from the noise they produce. Finally, a video camera (jAi, CM080GE) is used to acquire images of the bead from above and to determine its position in the horizontal plane as a function of time. The signals from the inductive sensor and microphone, as well as the images from the camera, are recorded during the whole experimental time and processed subsequently. The acquisition rates, $f_{s}=10 \mathrm{kHz}$ for the inductive sensor and the microphone and $f_{i}=20$ frames per second for the camera, ensure a sufficient temporal resolution while keeping a reasonable size of the data sets. 


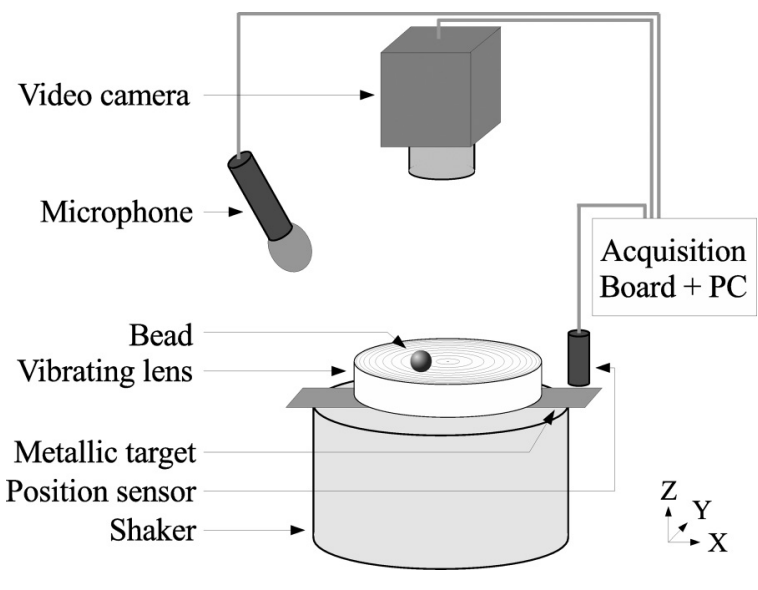

FIG. 1. Sketch of the experimental setup.

For the sake of simplicity, the amplitude $A$ and frequency $f$ of the vertical oscillation, $z(t)=A \sin (2 \pi f t)$, are chosen such that the bead is locked into the so-called mode 1 : the bead collides with the lens once per period $1 / f$. For a given restitution coefficient $e(\simeq 0.81$ in our experimental conditions) this is possible only if the reduced acceleration $\Gamma \equiv A(2 \pi f)^{2} / g$ is large enough for the mode 1 to exist $\left(\Gamma>\frac{1-e}{1+e} \pi \simeq 0.33\right)$ and small enough to avoid period doubling (in practice, $\Gamma<1$ ) [23].

\section{EXPERIMENTAL OBSERVATIONS AND FIRST ANALYSIS}

Whereas a concave surface is usually used as a trick to maintain the bead at the vertical of the vibrator axis, we observe strikingly that, even if it indeed does not escape from the system, the bead exhibits a significant and complex horizontal motion. We report in Fig. 2 a typical trajectory: the bead first moves aside and starts following an almost elliptic trajectory whose main axis slowly rotates. With time, the typical amplitude of the horizontal motion increases in both directions until an almost circular trajectory is reached in the steady state. This constitutes the main observation of the present study.

Before tackling the instability mechanism, we comment on the characteristic frequency, $f_{0}$, associated with the horizontal motion. First, $f_{0}$ is almost constant during the growth of the instability. Second, in the whole experimental range, we
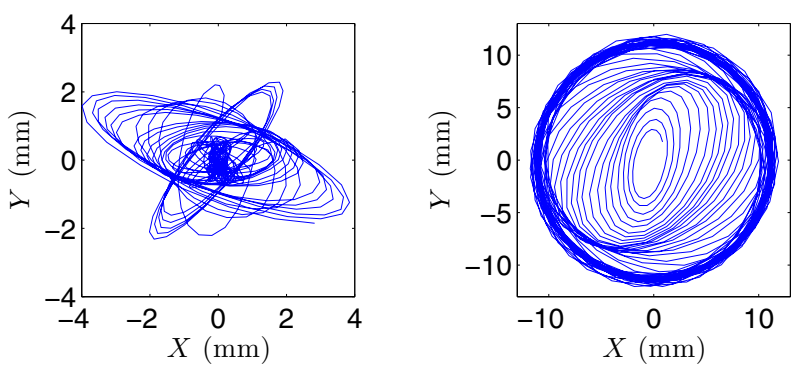

FIG. 2. (Color online) Typical trajectories of the bead in the horizontal $(X-Y)$ plane-early (left) and final (right) stages. $f=$ $30 \mathrm{~Hz}, A=4.110^{-2} \mathrm{~cm}, R=15.6 \mathrm{~cm}$, and $r=0.5 \mathrm{~cm}$. measured $f_{0}=(1.10 \pm 0.02) \mathrm{Hz}$, independent of the vibration frequency $f(\in[30 ; 70] \mathrm{Hz})$ and amplitude $A$. This observation can be understood using the following rough arguments: Let us assume that the bead moves only in a vertical plane containing the vertical axis of the shaker and consider the permanent regime. Since the bead is locked in mode 1 , the duration of the free fall between two successive collisions is $1 / f$ and its vertical velocity at the collision $V_{z}=-g /(2 f)$, where $z$ denotes the vertical coordinate. Supposing a Snell-Descartes reflection of the bead at the impact, the change in its horizontal velocity follows $\Delta V_{x}=2\left(\frac{x}{R}\right) V_{z}$, where $x$ denotes the distance to the center in the horizontal plane and, thus, $\frac{x}{R}$ the local slope the surface makes with the horizontal. Considering the fact that the bead collides with the surface with the frequency $f$, we write $\ddot{x}=f \Delta V_{x}$ and, then, $\ddot{x}=-\frac{g}{R} x$, where denotes the derivative with respect to time. Such simple arguments lead to $f_{0}=\frac{1}{2 \pi} \sqrt{g / R}$, the frequency of the pendulum of length $R$, thus independent of $f$ and $A$. Whereas the independence of $f_{0}$ on $A$ was not surprising as none of the characteristics of the motion depends on $A$ in mode 1 , the proposed arguments account for the independence of $f_{0}$ on $f$, which was not obvious. They lead to the theoretical value $f_{0}=1.26 \mathrm{~Hz}$, which is close to the experimental one, but there is a measurable difference between these two frequencies. Quantitative agreement was not expected as many experimental features were neglected by limiting the motion in a vertical plane, leaving out the spin of the bead around its own axis and the velocity of the surface at the collision, and making a strong assumption on the collision rule. But more importantly, our rough arguments do not account for any amplification mechanism that can explain the growth of an instability and finer analysis is needed.

Seeking for clues to help modeling the system, we performed a series of experimental tests. First, we checked by tilting the axis of the shaker that the instability was not driven by a misalignment of the vibration with the vertical. The only consequence of the tilt is an elliptical trajectory with the major axis oriented in the tilt direction. Second, we considered that a possible source of instability could be a modulation, resulting from the horizontal motion of the bead, of the time between two successive collisions. Indeed, moving away from the axis, the bead "sees" a higher surface and the free-fall time is reduced. We checked that such modulation has no or little effect by imposing, separately, slow (with frequencies of the order of $f_{0}$ ) variations (of about 10\%) of the frequency $f$ and of the amplitude $A$ of the vertical motion. We did not observe any significant change in the behavior of the system. Third, we performed experiments with beads of different sizes $(r=0.1$ to $1 \mathrm{~cm})$ and made of different material (stainless steal, plastic, cork). The instability is always observed, but we did not manage to study separately the influence of the bead size and mass and of the properties of the contact (restitution, friction). Finally, to better characterize the growth of the instability, we measured the distance to the center $D(t)$ as function of time $t$ (Fig. 3) from which we estimated the growth rate $\sigma$ and steady-state radius of the trajectory $D_{\max }$ [Fig. 4, $\sigma$ is obtained by adjusting the local maxima of $D(t)$ in Fig. 3 to $\exp (\sigma t)$ ]. The amplitude $A$ of vibration alters neither $\sigma$ nor $D_{\max }$ (not shown), as long as the bead remains locked in mode 1 . By contrast, the growth rate $\sigma$ and $D_{\max }$ significantly decrease when $f$ is increased. 


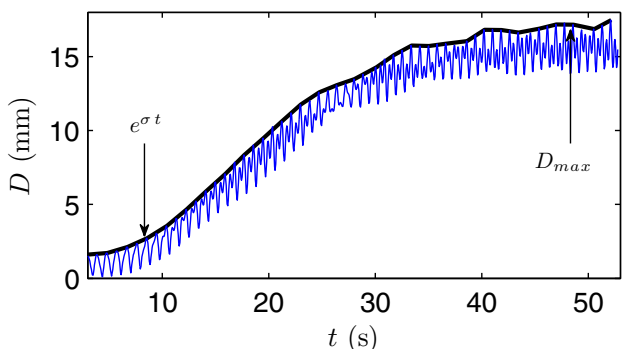

FIG. 3. (Color online) Typical behavior of the distance to the center $D(t)$. The initial, exponential growth of the instability with the rate $\sigma$ is observed and the late saturation toward $D(\infty) \equiv D_{\max }$. $f=30 \mathrm{~Hz}, A=4.010^{-2} \mathrm{~cm}, R=15.6 \mathrm{~cm}$, and $r=0.5 \mathrm{~cm}$.

\section{2D MODEL}

To account for the instability, and for the decrease of $\sigma$ and $D_{\max }$ with $f$, the problem is considered in $2 \mathrm{D}$, in a vertical plane containing the axis of the shaker. The frame of reference is attached to the vibrating surface and the vertical axis is oriented downwards (Fig. 5). The spin of the bead in the horizontal plane (with the angular velocity $\Omega$, a signed scalar) is taken into account and the collisions are accounted for more precisely than previously. In the moving frame, in addition to any external force $\mathbf{f}$, the additional acceleration due to the motion of the frame $-m A \omega^{2} \sin (\omega t) \mathbf{i}$ must be taken into account ( $m$ denotes the mass of the bead and $\mathbf{i}$ the unit vector along the vertical). The problem is considered in polar coordinates $\rho$ and $\theta$ (Fig. 5) and the characteristic frequency of the pendulum $v \equiv \sqrt{g /(R-r)}$ is introduced for convenience ( $R-r$ is indeed the distance between the center of curvarture of the lens and the center of mass of the bead). The bead motion has two phases: the free fall between collisions and the collisions.

The free fall is the easiest to account for since $\mathbf{f}=m g \mathbf{i}$ and $\dot{\Omega}=0$. In the radial component $\ddot{\rho}-\rho \dot{\theta}^{2}$ of the bead acceleration, the second term can be neglected as $\ddot{\rho}$ is of the order of $g$ and $\rho \dot{\theta}^{2}$ of the order of $g(v / f)^{2} \Delta \theta^{2}$. Indeed, the characteristic frequency of the pendulum $v$ is much smaller than the vibration frequency $f$ and the angular amplitude $\Delta \theta$ of the oscillations is less or about unity. The dynamical equation for $\rho(t)$, which reduces to $\ddot{\rho}=g[1-\Gamma \sin (\omega t)]$, can be solved analytically. With $n$ the index of the collision and the assumption that the duration of the free fall is $1 / f$ (i.e., $t_{n+1}=t_{n}+1 / f$ ), the boundary conditions reads
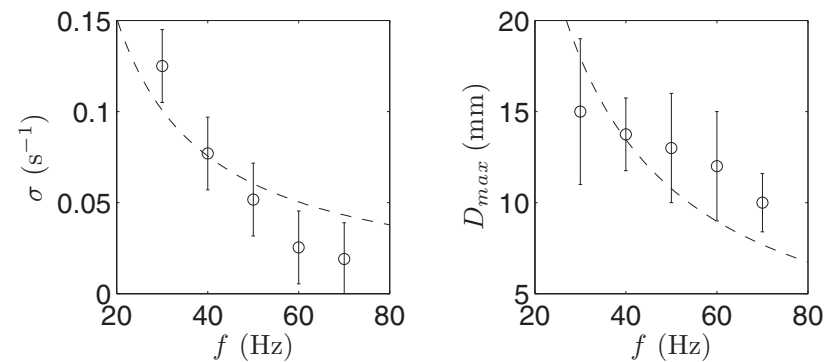

FIG. 4. Left: Growth rate $\sigma$ as a function of $f$; right: $D_{\max }$ as a function of $f$ (circles, average over $\sim 10$ realizations; dashed lines, fit $\left.\propto f^{-1}\right) . R=15.6 \mathrm{~cm}$ and $r=0.5 \mathrm{~cm}$.

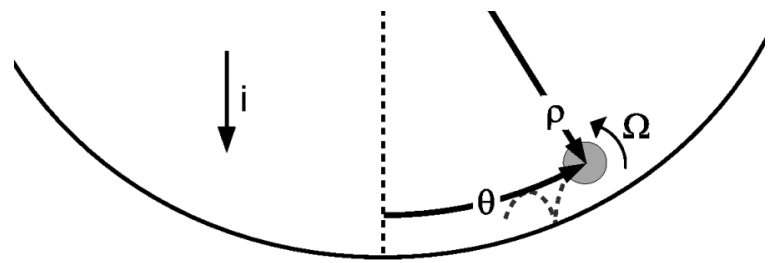

FIG. 5. Definition of the variables $\rho, \theta$, and $\Omega$ in the model.

$\rho\left(t_{n}\right)=R-r$ and $\rho\left(t_{n}+1 / f\right)=R-r$. From now on, a - sign is used to indicate a quantity prior to the collision and $a+$ sign to indicate a quantity after the collision. Note that the solution for the free fall imposes a first relation between $\dot{\rho}_{n}^{+} \equiv \dot{\rho}\left(t_{n}\right)$ and $\dot{\rho}_{n+1}^{-} \equiv \dot{\rho}\left(t_{n}+1 / f\right)$. In addition, at collision $n$, provided that the bead impinges with the normal velocity $\dot{\rho}_{n}^{-}$and takes off with the normal velocity $\dot{\rho}_{n}^{+}$, the collision law reads

$$
\dot{\rho}_{n}^{+}=-e \dot{\rho}_{n}^{-},
$$

where $e$ is the restitution coefficient. With the additional assumption that the radial motion is periodic, the collision time $t_{n}$ obeys the classical relation $\cos \left(2 \pi f t_{n}\right)=\frac{1-e}{1+e} \frac{\pi}{\Gamma}$. Then, the dynamical equation in the radial direction links $\theta(t)$ and $\rho(t)$ and, in the limit $f \gg v$,

$$
\dot{\theta}_{n+1}^{-}=\left[1-\frac{1}{1+e}\left(\frac{v}{f}\right)^{2}\right] \dot{\theta}_{n}^{+}-\left(\frac{v}{f}\right) \theta_{n}^{+} .
$$

The collision is more difficult to account for, and additional assumptions concerning the collision law are needed. At contact, the bead is subjected to the contact force, which is decomposed in its normal (radial) $N$ and its tangential $T$ components. First, an estimate of $N$ is obtained by introducing the duration of the collision $\tau$ and writing, for collision $n$, $\ddot{\rho}=\frac{\dot{\rho}_{n}^{+}-\dot{\rho}_{n}^{-}}{\tau}$, and, then, $N \simeq m \ddot{\rho}$ (in the limit $\tau \rightarrow 0$, the acceleration due to gravity is negligible during the collision). The use of Eq. (1) leads to $N=-m(1+e) \frac{\dot{\rho}_{n}^{-}}{\tau}$. The dynamics of the bead at the collision is also governed by two additional equations: The equation governing the orthoradial velocity,

$$
m(R-r) \ddot{\theta}=T-m g[1-\Gamma \sin (2 \pi f t)] \sin (\theta),
$$

and the dynamical equation coupling the torque $r T$ and the angular momentum $J \Omega$,

$$
J \dot{\Omega}=r T,
$$

where $J=\frac{2}{5} m r^{2}$ for a bead. Equations (3) and (4) both involve the tangential component of the contact force $T$ and additional assumptions are needed to go farther. Since the bead is initially at the center of the lens, bouncing mostly vertically with no spin, the tangential component $T$ is likely negligible compared to the normal one $N$. Consequently, it is reasonable to consider that the contact point, $I$, is not sliding and that its velocity $v_{I}=r \Omega+(R-r) \dot{\theta}$ is initially governed by a collision rule similar to Eq. (1):

$$
v_{I_{n}}^{+}=e^{\prime} v_{I_{n}^{-}}^{-} .
$$

Thus, a second restitution coefficient, $e^{\prime}$, is introduced here, which potentially differs from $e$ [24]. Equations (3), (4), and (5) 
lead to

$$
\begin{aligned}
& \Omega_{n}^{+}=\frac{2+5 e^{\prime}}{7} \Omega_{n}^{-}+\frac{5\left(1-e^{\prime}\right)}{7} \frac{R-r}{r} \dot{\theta}_{n}^{-}, \\
& \dot{\theta}_{n}^{+}=\frac{5+2 e^{\prime}}{7} \dot{\theta}_{n}^{-}+\frac{2\left(1-e^{\prime}\right)}{7} \frac{r}{r-R} \Omega_{n}^{-} .
\end{aligned}
$$

The collision is thus accounted for by Eqs. (1), (6), and (7), which give the value of dynamical quantities after the collision, $\dot{\rho}_{n}^{+}, \Omega_{n}^{+}$, and $\dot{\theta}_{n}^{+}$, as functions of their values prior to the collision, $\dot{\rho}_{n}^{-}, \Omega_{n}^{-}$, and $\dot{\theta}_{n}^{-}$.

From now on, successive collisions are considered. Equation (2), $\theta_{n+1}^{-}=\theta_{n}^{+}+\dot{\theta}_{n}^{+} / f$, and $\Omega_{n+1}^{-}=\Omega_{n}^{+}$account for the variations in $\theta, \dot{\theta}$, and $\Omega$ during the free fall between collisions $n$ and $n+1$. Then, using Eqs. (1), (6), and (7), and defining $\epsilon \equiv r /(R-r)$, a recurrence relation for the vector $\mathbf{X}_{n}=\left(\epsilon \Omega_{n}^{+}, \dot{\theta}_{n}^{+}, \theta_{n}^{+}\right)$is obtained in the form $\mathbf{X}_{n+1}=\mathcal{M} \mathbf{X}_{n}$, where

$$
\mathcal{M}=\left[\begin{array}{ccc}
\frac{\left(2+5 e^{\prime}\right)}{7} & \frac{5\left(1-e^{\prime}\right)}{7}\left[1-\frac{v^{2}}{(1+e) f^{2}}\right] & -\frac{5\left(1-e^{\prime}\right)}{7} \frac{v}{f} \\
\frac{2\left(1-e^{\prime}\right)}{7} & \frac{\left(5+2 e^{\prime}\right)}{7}\left[1-\frac{v^{2}}{(1+e) f^{2}}\right] & -\frac{\left(5+2 e^{\prime}\right)}{7} \frac{v}{f} \\
0 & \frac{v}{f} & 1
\end{array}\right]
$$

From the eigenvalues of $\mathcal{M}$, an oscillation at the frequency,

$$
f_{0}=\frac{1}{2 \pi} \sqrt{\frac{5}{7}} v+\mathcal{O}\left(\frac{v}{f}\right),
$$

can grow with the rate

$$
\sigma=\frac{5 e-2 e^{\prime}-7 e e^{\prime}}{14(1+e)\left(1-e^{\prime}\right)} \frac{v^{2}}{f}+\mathcal{O}\left(\frac{v^{2}}{f^{2}}\right),
$$

where, we recall, $v \equiv \sqrt{g /(R-r)}$. From the experimental parameters, Eq. (9) leads to $f_{0}=(1.095 \pm 0.005) \mathrm{Hz}$ in excellent agreement with the experimental value. In addition, $\sigma$ is predicted to decrease for increasing $f$ as observed experimentally (Fig. 4). The fit to the experimental data leads to the reasonable estimate $e^{\prime} \approx 0.45$ [24].

The model predicts an unlimited exponential growth of the amplitude of the pendular motion [Eq. (10)] and thus of the particle energy as result of the successive collisions with the massive substrate. The phenomenon reminds of the so-called Fermi acceleration [25]. Such divergence of the energy is usually impeded by dissipation (for the classical bouncing ball, with one degree of freedom, by a restitution coefficient less than unity) [26,27]. One can, however, suggest that, in our experimental configuration, the growth of the instability is likely limited by a change in the collision rule. Indeed, the bead can slide during the collision when it departs significantly from center. This suggestion is supported by the observation that, qualitatively, beads exhibiting a larger friction experience a motion of larger amplitude. Indeed, for large value of $\theta,|T / N|$ can overcome the friction coefficient $\mu$ and the bead starts sliding. From the dynamics of the bead, one can estimate $T / N$. From Eq. (1), $N=-m(1+e) \frac{\dot{\rho}_{n}^{-}}{\tau}$. From Eq. (5), $T=\frac{2}{7 \tau} \frac{1-e^{\prime}}{e^{\prime}} m\left[r \Omega_{n}^{+}+(R-r) \dot{\theta}_{n}^{+}\right]$. Considering a periodic solution for $\theta(t)$ at the frequency $f_{0}$, one obtains that the onset of sliding is reached for the amplitude:

$$
D_{\max } \simeq \frac{49 \pi}{20} \frac{(1+e) e^{\prime}}{1-e^{\prime}}\left(\frac{f_{0}}{f}\right) \mu R .
$$

Even if the agreement with the experiments is questionable, in particular the dependency of $D_{\max }$ on $f$, the quantitative comparison with the data reported in Fig. 4 leads to $\mu \approx 0.3$, which is also a reasonable value.

\section{DISCUSSION AND CONCLUSION}

The agreement with the experiments is only qualitative probably because results of a two-dimensional (2D) model are compared with experimental results of a three-dimensional (3D) experiment. We attempted to perform the experiment in $2 \mathrm{D}$, by confining the bead between two vertical walls. The experiments are delicate, and probably because of excessive friction with the lateral walls, we did not observe any significant in-plane motion of the bead. From the theoretical point of view, a 3D version of the model is probably accessible, but an excellent agreement with the experiments would require including a better description of the collision, which is still a debated problem nowadays [24,28]. Due to the sensitivity of the instability to the contact law, it might be interesting to simulate the problem numerically to test the laws commonly used in codes based on the discrete element method (DEM) [29,30].

In conclusion, the dynamics of a bead bouncing upon a curved, vertically vibrated surface reveals an unexpected and intriguing instability. Locked in the first periodic mode, the bead experiences a pendular motion whose typical frequency $f_{0}$ depends neither on the amplitude nor on the frequency of the surface vibration, but only on the distance $R-r$ between the center of mass of the bead and the center of curvature of the surface. We reported the first experimental evidence of the phenomenon and proposed a theoretical approach that reveals the underlying mechanisms. The instability grows due to the coupling between the pendular motion and the spin of the bead around an horizontal axis, which is insured by a nonsliding contact at the collision. Strikingly, the growth rate decreases when the frequency of the vibration is increased. The growth of the instability is limited by the sliding at the contact during the collision, thus by friction.
[1] H. M. Jaeger, S. R. Nagel, and R. P. Behringer, Granular solids, liquids, and gases, Rev. Mod. Phys. 68, 1259 (1996).

[2] J. B. Knight, C. G. Fandrich, C. N. Lau, H. M. Jaeger, and S. R. Nagel, Density relaxation in a vibrated granular material, Phys. Rev. E 51, 3957 (1995).
[3] P. Philippe and D. Bideau, Compaction dynamics of a granular medium under vertical tapping, Europhys. Lett. 60, 677 (2002).

[4] P. Richard, M. Nicodemi, R. Delannay, P. Ribière, and D. Bideau, Slow relaxation and compaction of granular systems, Nat. Mater. 4, 121 (2005). 
[5] J. B. Knight, E. E. Ehrichs, V. Y. Kuperman, J. K. Flint, H. M. Jeager, and S. R. Nagel, Experimental study of granular convection, Phys. Rev. E 54, 5726 (1996).

[6] J. B. Knight, H. M. Jeager, and S. R. Nagel, Vibration induced separation in granular media: The convection connection, Phys. Rev. Lett. 70, 3728 (1993).

[7] A. Kudrolli, Size separation in vibrated granular matter, Rep. Prog. Phys. 67, 209 (2004).

[8] F. Melo, P. B. Umbanhowar, and H. L. Swinney, Hexagons, kinks, and disorder in oscillated granular layers, Phys. Rev. Lett. 75, 3838 (1995).

[9] P. B. Umbanhowar, F. Melo, and H. L. Swinney, Localized excitations in a vertically vibrated granular layer, Nature 382, 793 (1996).

[10] V. Kumaran, Temperature of a granular material "fluidized" by external vibrations, Phys. Rev. E 57, 5660 (1998).

[11] A. Naert, Experimental study of work exchange with a granular gas: The viewpoint of the Fluctuation Theorem, Europhys. Lett. 97, 20010 (2012).

[12] W. Losert, D. G. W. Cooper, J. Delour, A. Kudrolli, and J. P. Gollub, Velocity statistics in excited granular media, Chaos $\mathbf{9}$, 682 (1999).

[13] J. S. Olafsen and J. S. Urbach, Clustering, order, and collapse in a driven granular monolayer, Phys. Rev. Lett. 81, 4369 (1998).

[14] M. G. Clerc, P. Cordero, J. Dunstan, K. Huff, N. Mujica, D. Risso, and G. Varas, Liquid-solid-like transition in quasi-onedimensional driven granular media, Nat. Phys. 4, 249 (2008).

[15] V. Narayan, N. Menon, and S. Ramaswamy, Nonequilibrium steady states in a vibrated-rod monolayer: Tetratic, nematic, and smectic correlations, J. Stat. Mech. (2006) P01005.

[16] S. Ramaswamy, The mechanics and statistics of active matter, Annu. Rev. Condens. Matter Phys. 1, 323 (2010).

[17] P. Pieránski, Jumping particle model. Period doubling cascade in an experimental system, J. Physique 44, 573 (1983).

[18] J. M. Luck and A. Mehta, Bouncing ball with a finite restitution-Chattering, locking and chaos, Phys. Rev. E 48, 3988 (1993).
[19] E. D. Leonel and A. L. P. Livorati, Thermodynamics of a bouncer model: A simplified one-dimensional gas, Commun. Nonlinear Sci. Numer. Simulat. 20, 159 (2015).

[20] S. Warr, W. Cooke, R. C. Balla, and J. M. Huntley, Probability distribution functions for a single-particle vibrating in one dimension: Experimental study and theoretical analysis, Physica A 231, 551 (1996).

[21] J.-C. Géminard and C. Laroche, Energy of a single bead bouncing on a vibrating plate: Experiments and numerical simulations, Phys. Rev. E 68, 031305 (2003).

[22] H. S. Wright, M. R. Swift, and P. J. King, The horizontal stability of a ball bouncing upon a vertically vibrated concave surface, Europhys. Lett. 81, 14002 (2008).

[23] J. J. Barroso, M. V. Carneiro, and E. E. N. Macau, Bouncing ball problem: Stability of the periodic modes, Phys. Rev. E 79, 026206 (2009).

[24] A. Doménech-Carbó, On the tangential restitution problem: Independent friction-restitution modeling, Gran. Matter 16, 573 (2014).

[25] M. A. Lieberman and A. J. Lichtenberg, Stochastic and adiabatic behavior of particles accelerated by periodic forces, Phys. Rev. A 5, 1852 (1972).

[26] André Luis Prando Livorati, D. G. Ladeira, and E. D. Leonel, Scaling investigation of Fermi acceleration on a dissipative bouncer model, Phys. Rev. E 78, 056205 (2008).

[27] E. D. Leonel and A. L. P. Livorati, Describing Fermi acceleration with a scaling approach: The Bouncer model revisited, Physica A 387, 1155 (2008).

[28] C. Thornton, S. J. Cummins, and P. W. Cleary, An investigation of the comparative behavior of alternative contact force models during inelastic collisions, Powder Tech. 233, 30 (2013).

[29] P. A. Cundall and O. D. L. Strack, Discrete numerical model for granular assemblies, Geotechnique 29, 47 (1979).

[30] J. Schaefer, S. Dippel, and D. Wolf, Force schemes in simulations of granular materials, J. Phys. I France 6, 5 (1996). 